Alberta-Thy-18-97

hep-th/9709074

\title{
Finite Temperature Nonlocal Effective Action for Scalar Fields
}

\author{
Yuri Gusev' \\ Theoretical Physics Institute, University of Alberta \\ Edmonton, Alberta, Canada T6G 2J1 \\ Andrei Zelnikovf \\ Theoretical Physics Institute, University of Alberta \\ Edmonton, Alberta, Canada T6G 2J1 \\ and \\ P.N. Lebedev Physics Institute \\ Leninskii prospect 53, Moscow 117 924 Russia
}

\begin{abstract}
Scalar fields at finite temperature are considered in four dimensional ultrastatic curved spacetime. One loop nonlocal effective action at finite temperature is found up to the second order in curvature expansion. This action is explicitly infrared finite. In the high temperature expansion of free energy, essentially nonlocal terms linear in temperature are derived.
\end{abstract}

\footnotetext{
1e-mail: ygusev@phys.ualberta.ca

2e-mail: zelnikov@phys.ualberta.ca
} 


\section{Introduction}

Effective action is a powerful tool in quantum field theory. It contains information about all Green functions, vacuum polarization effects, etc. In general case it is a non-local functional. To study thermodynamic properties of a system one has to know the effective action at nonzero temperature. In this letter we calculate the one loop nonlocal effective action and free energy for quantum scalar fields $\varphi$ in a curved spacetime at finite temperature $T$. At first we introduce general notations for both zero and finite temperature theories on some $D$-dimensional manifold. We consider fields $\varphi$ that satisfy the equation

$$
F(\nabla) \varphi=0, \quad F(\nabla)=\square+P(x)-\frac{1}{6} R(x) .
$$

Here the Laplace-Beltrami operator $\square \equiv g^{\mu \nu} \nabla_{\mu} \nabla_{\nu}$ is constructed in terms of a covariant derivative $\nabla_{\mu}$. The metric $g^{\mu \nu}$ is characterized by its Riemann and Ricci curvatures $R_{\cdot \alpha \nu \beta}^{\mu}=$ $\partial_{\nu} \Gamma_{\alpha \beta}^{\mu}-\ldots, \quad R_{\alpha \beta}=R_{\cdot \alpha \mu \beta}^{\mu}$. The term with scalar curvature $R / 6$ in the operator $F(\nabla)$ is explicitly singled out for convenience only. The potential $P(x)$ has an arbitrary structure and may include a curvature $R(x)$ or an interaction potential. This means that models satisfying eq. (1) are quite general and describe non-conformal and interacting scalar fields.

The one loop effective action can be expressed in terms of eigen values of the operator $F(\nabla)$ and formally reads $W=\frac{1}{2} \operatorname{Tr} \ln F$. It can be written [1, 2] in the form of an integral over the proper time $s$,

$$
W=-\frac{1}{2} \int_{0}^{\infty} \frac{\mathrm{d} s}{s} \operatorname{Tr} K(s) .
$$

In coordinate representation $\operatorname{Tr}$ denotes the functional trace $\operatorname{Tr} K(s)=\int \mathrm{d}^{D} x K(s \mid x, x)$ of the heat kernel, which is defined as a solution of the equations

$$
\frac{\mathrm{d}}{\mathrm{d} s} K(s \mid x, y)=F\left(\nabla^{x}\right) K(s \mid x, y), \quad K(0 \mid x, y)=\delta(x-y) .
$$

Knowledge of the heat kernel with separated points allows one to construct the covariant diagrammatic expansion of the effective action to all loop orders [1], 2]. For our case of one loop computations, it is sufficient to find the trace of the heat kernel.

Thermodynamics of a system is well defined if background fields are static, so we restrict ourselves to considering static spacetimes. It is well known [3, 4, 5] that a quantum field theory defined in a Euclidean spacetime, which is periodic (for bosons) in imaginary time $\tau$ with a period $\beta$, amounts to a thermal field theory with temperature $T=1 / \beta$. In this letter we consider Euclidean manifolds with the topology of a cylinder $S^{1} \times R^{3}$. The periodic (thermal) heat kernel $K^{\beta}$ can be expressed as an infinite sum of zero temperature (vacuum) heat kernels [5, 6]

$$
K^{\beta}\left(s \mid \tau, \boldsymbol{x} ; \tau^{\prime}, \boldsymbol{x}^{\prime}\right)=\sum_{n=-\infty}^{\infty} K\left(s \mid \tau, \boldsymbol{x} ; \tau^{\prime}+\beta n, \boldsymbol{x}^{\prime}\right)
$$

This image sum is equivalent to summation over Matsubara frequencies in a momentum space representation in thermal field theory. 
We restrict now a class of spacetimes to the one possessing ultrastatic (optical) metrics

$$
\mathrm{d} s^{2}=g_{\mu \nu} \mathrm{d} x^{\mu} d x^{\nu}=\mathrm{d} \tau^{2}+\bar{g}_{i j}(\boldsymbol{x}) \mathrm{d} x^{i} \mathrm{~d} x^{j}
$$

where $\mu, \nu=(0,1,2,3)$ and $i, j=(1,2,3)$, and the metric tensor $\bar{g}_{i j}(\boldsymbol{x})$ depends on the spatial coordinates $\boldsymbol{x}$ only. To take into account temperature effects in curved spacetime explicitly one has to factorize the heat kernel into temporal and spatial $K^{(3)}(s)$ parts,

$$
\bar{K}\left(s \mid \tau, \boldsymbol{x} ; \tau^{\prime}, \boldsymbol{x}^{\prime}\right)=\frac{1}{(4 \pi s)^{1 / 2}} \exp \left(-\frac{\left(\tau-\tau^{\prime}\right)^{2}}{4 s}\right) \bar{K}^{(3)}\left(s \mid \boldsymbol{x} ; \boldsymbol{x}^{\prime}\right),
$$

what is possible to do in ultrastatic spacetimes. Here and below we denote objects in ultrastatic spacetimes by overbars. Then, the trace of (4) takes an elegant form [7, 8] in terms of Riemann theta function, which is defined in a usual way $\theta_{3}(a, b)=\sum_{n=-\infty}^{n=\infty} \mathrm{e}^{2 n a \mathrm{i}} b^{n^{2}}$,

$$
\operatorname{Tr} \bar{K}^{\beta}(s)=\theta_{3}\left(0, \mathrm{e}^{-\frac{\beta^{2}}{4 s}}\right) \frac{\beta}{(4 \pi s)^{1 / 2}} \int \mathrm{d}^{3} \boldsymbol{x} \bar{K}^{(3)}(s \mid \boldsymbol{x}, \boldsymbol{x}) .
$$

\section{Nonlocal effective action and free energy at finite temperature}

Various methods are applicable for calculation of $\operatorname{Tr} \bar{K}$. A traditional tool of quantum field theory in curved spacetime, the Schwinger-DeWitt series [1, 2], corresponding to the heat kernel expansion at small proper times $s$, is useful only for obtaining high temperature expansion of effective action [7]. We resort to a method of covariant perturbation theory [9, 10, 11, 12], since local representations for the heat kernel are not adequate to our problem. This technique, as proposed by Vilkovisky in ref. [13], is a regular procedure for covariant expansion of $K(s)$ in powers of curvatures (field strengths). This is essentially nonlocal representation, and it takes into account an infinite number of derivatives acting on curvatures. At small $s$ it reproduces the local Schwinger-DeWitt expansion [10, 14].

Besides, the Schwinger-DeWitt expansion, which is very effective in dealing with ultraviolet divergencies of the effective action [2], fails in the infrared (large $s$ ) regime for massless theories. In contrast, the one loop effective action and all Green functions computed in the framework of covariant perturbation theory are infrared finite if the dimension of a manifold under consideration is larger than two [9, 10, 15, 14. In this letter we show that the one loop nonlocal effective action for arbitrary scalar fields calculated on a four dimensional manifold remains infrared finite at finite temperature. This fact is very important, for infrared divergencies are one of major obstacles in thermal field theory [16].

It is necessary now to give a brief description of covariant perturbation theory at zero temperature in arbitrary dimensions $D$. Let us first display a structure of the nonlocal trace of the heat kernel [10",

$$
\begin{aligned}
\operatorname{Tr} K(s)= & \frac{1}{(4 \pi s)^{D / 2}} \int \mathrm{d}^{D} x g^{1 / 2}\left\{1+s P+s^{2}\left[R_{\mu \nu} f_{1}(-s \square) R^{\mu \nu}\right.\right. \\
& \left.\left.+R f_{2}(-s \square) R+P f_{3}(-s \square) R+P f_{4}(-s \square) P\right]\right\}+\mathrm{O}\left[\Re^{3}\right] .
\end{aligned}
$$


Form factors $f_{i}$ are analytic functions of the dimensionless argument $s \square$. They act on tensor invariants constructed of the set of field strengths $R^{\alpha \beta}, P$ (called here curvatures) characterizing background. We use for these curvatures the collective notation $\Re$. First two terms of the sum (8) are purely local and coincide with first two coefficients of the short time expansion [10]. The Euclidean spacetime is supposed to be asymptotically flat and have the topology $R^{D}$. Therefore, gravitational curvatures and potential $P$ should vanish at infinity [10]. The calculations in covariant perturbation theory are carried out with accuracy $\mathrm{O}\left[\Re^{n}\right]$, i.e., up to terms of $n$-th and higher power in the curvature $\Re$. Thus, this curvature expansion is valid for highly inhomogeneous background fields, $\nabla \nabla \Re>>\Re^{2}$.

All form factors in (8) can be expressed in terms of one basic form factor

$$
f(-s \square)=\int_{0}^{1} \mathrm{~d} \alpha \mathrm{e}^{\alpha(1-\alpha) s \square} .
$$

Their explicit form reads [10]

$$
\begin{aligned}
f_{1}(-s \square) & =\frac{f(-s \square)-1-\frac{1}{6} s \square}{(s \square)^{2}}, \\
f_{2}(-s \square) & =\frac{1}{8}\left[\frac{1}{36} f(-s \square)-\frac{1}{3} \frac{f(-s \square)-1}{s \square}-\frac{f(-s \square)-1-\frac{1}{6} s \square}{(s \square)^{2}}\right], \\
f_{3}(-s \square) & =\frac{1}{12} f(-s \square)-\frac{1}{2} \frac{f(-s \square)-1}{s \square}, \\
f_{4}(-s \square) & =\frac{1}{2} f(-s \square) .
\end{aligned}
$$

Let us return now to the finite temperature case. The free energy for scalar fields in ultrastatic spacetime $\bar{F}^{\beta}$ is defined in terms of the Euclidean effective action at finite temperature $\bar{W}^{\beta}$ by the relation

$$
\bar{F}^{\beta} \equiv \frac{1}{\beta} \bar{W}^{\beta}=-\frac{1}{2 \beta} \int_{0}^{\infty} \frac{\mathrm{ds}}{s} \operatorname{Tr} \bar{K}^{\beta}(s) .
$$

It is convenient to separate a vacuum mode $n=0$ from the infinite sum in (偌), since only vacuum effective action contains ultraviolet divergencies [17]. This is equivalent to subtracting the zero temperature free energy $\bar{F}^{\infty}$ from $\bar{F}^{\beta}$. Since only renormalized effective action and free energy have physical meaning, we renormalize one loop vacuum effective action (2) with the use of zeta function regularization:

$$
\bar{W}_{\mathrm{ren}}^{\infty}=-\frac{1}{2} \frac{\partial}{\partial \epsilon}\left[\frac{\mu^{2 \epsilon}}{\Gamma(\epsilon)} \int_{0}^{\infty} \frac{\mathrm{d} s}{s^{1-\epsilon}} \operatorname{Tr} \bar{K}^{\infty}(s)\right]_{\epsilon=0},
$$

where $\mu$ is a mass-like regularization parameter and $\Gamma$ is the gamma function. $\bar{F}_{\text {ren }}^{\infty}$ will be combined with $n \neq 0$ terms at the end of our derivations. Thus, we compute

$$
\bar{F}_{\text {ren }}^{\beta}-\bar{F}_{\text {ren }}^{\infty}=-\frac{1}{2} \int_{0}^{\infty} \frac{\mathrm{d} s}{s}\left(\Theta_{3}\left(0, \mathrm{e}^{-\frac{\beta^{2}}{4 s}}\right)-1\right) \frac{1}{(4 \pi s)^{1 / 2}} \int \mathrm{d}^{3} x \bar{K}^{(3)}(s \mid \boldsymbol{x} ; \boldsymbol{x}) .
$$

The three dimensional heat kernel $\bar{K}^{(3)}(s)$ is defined as a solution of (3) with the three dimensional operator (1). In this case the operator $\square$ is the three dimensional Laplacian 
$\triangle$, and the curvatures $\bar{\Re}=\left(\bar{P}(\boldsymbol{x}), \bar{R}_{i j}(\boldsymbol{x})\right)$ are defined on a three dimensional hypersurface $\tau=$ const.

The integral (13) with first two terms of the trace of the heat kernel (8) gives just numerical coefficients. This local contribution to free energy is known [8, 17] and coincides with first two terms of high temperature expansion [18]. The problem reduces to computation of the thermal form factors

$$
\gamma_{i}^{\beta}(-\triangle)=\int_{0}^{\infty} \frac{\mathrm{d} y}{y}\left[\theta_{3}\left(0, \mathrm{e}^{-y}\right)-1\right] f_{i}\left(-\frac{\beta^{2}}{4 y} \triangle\right),
$$

where $y=\beta^{2} / 4 s$.

Calculation of (14) is a key technical part of this letter and we explain its idea on the example of the basic form factor (9). Straightforward integration over $y$ leaves us with the modified Bessel function of the second kind

$$
\gamma^{\beta}(-\triangle)=4 \sum_{n=1}^{\infty} \int_{0}^{1} \mathrm{~d} \alpha K_{0}(n \beta \sqrt{\alpha(1-\alpha)} \sqrt{-\triangle}) .
$$

Another change of variables, $x=2 \sqrt{\alpha(1-\alpha)}$, allows us to find its form in terms of the exponential integrals,

$$
\int_{0}^{1} \mathrm{~d} x \frac{x}{\sqrt{1-x^{2}}} K_{0}\left(\frac{n z x}{2}\right)=\frac{1}{n z}\left[\operatorname{Ei}\left(\frac{n z}{2}\right) \mathrm{e}^{-n z / 2}-\operatorname{Ei}\left(-\frac{n z}{2}\right) \mathrm{e}^{n z / 2}\right],
$$

where $z=\beta \sqrt{-\triangle}$. Now we can use for the right hand side of (16) its standard form in terms of elementary functions and obtain

$$
\gamma^{\beta}(-\triangle)=4 \int_{0}^{\infty} \mathrm{d} t \sum_{n=1}^{\infty} \frac{\sin (t)}{t^{2}+(n z)^{2} / 4} .
$$

This is just what we need since the sum over $n$ can be exactly evaluated,

$$
\sum_{n=1}^{\infty} \frac{1}{t^{2}+n^{2} z^{2} / 4}=\frac{1}{2}\left[\frac{2 \pi}{z t} \frac{1}{\operatorname{th}(2 \pi t / z)}-\frac{1}{t^{2}}\right] .
$$

There are, as seen from (11), two other types of basic thermal form factors with one and two subtractions. Derivations for them are similar but more technically involved.

Finally, we arrive at the following integral representation,

$$
\begin{aligned}
\bar{F}_{\text {ren }}^{\beta}-\bar{F}_{\text {ren }}^{\infty}= & -\frac{1}{32 \pi^{2}} \int \mathrm{d}^{3} x \bar{g}^{1 / 2}\left\{\frac{16}{45} \frac{\pi^{4}}{\beta^{4}}+\frac{4}{3} \frac{\pi^{2}}{\beta^{2}} \bar{P}\right. \\
& +\left[\bar{R}_{i j} \gamma_{1}^{\beta}(-\triangle) \bar{R}^{i j}+\bar{R} \gamma_{2}^{\beta}(-\triangle) \bar{R}\right. \\
& \left.\left.+\bar{P} \gamma_{3}^{\beta}(-\triangle) \bar{R}+\bar{P} \gamma_{4}^{\beta}(-\triangle) \bar{P}\right]+\mathrm{O}\left[\bar{\Re}^{3}\right]\right\} .
\end{aligned}
$$

Here the thermal form factors have a form

$$
\gamma_{i}^{\beta}(-\triangle)=\int_{0}^{\infty} \mathrm{d} t g_{i}(t)\left[\frac{2 \pi}{z t} \frac{1}{\operatorname{th}(2 \pi t / z)}-\frac{1}{t^{2}}\right],
$$


and $g_{i}(i=1, \ldots 4)$ are the simple combinations of elementary functions

$$
\begin{aligned}
& g_{1}(t)=-\frac{1}{2}\left(\frac{\sin (t)}{t^{2}}+3\left[\frac{\cos (t)}{t^{3}}-\frac{\sin (t)}{t^{4}}\right]\right) \\
& g_{2}(t)=\frac{1}{48}\left(\frac{1}{3} \sin (t)+2 \frac{\cos (t)}{t}+\frac{\sin (t)}{t^{2}}+9\left[\frac{\cos (t)}{t^{3}}-\frac{\sin (t)}{t^{4}}\right]\right) \\
& g_{3}(t)=\frac{1}{2}\left(\frac{1}{3} \sin (t)+\frac{\cos (t)}{t}-\frac{\sin (t)}{t^{2}}\right) \\
& g_{4}(t)=\sin (t) .
\end{aligned}
$$

We have achieved our goal of evaluating the image sum (41), moreover the answer is expressed in terms of elementary functions. As can be seen from eq. (20) 'a golden rule' of field theory calculations [13], namely, to keep a proper time integration to the very end, has been also satisfied, as the variable $t$ (which is dimensionless in contrast to the proper time $s$ ) clearly plays a role of the proper time. One can check that integrals of this type are regular, and two apparent singularities at the lower limit compensate each other. The price for all this is that an argument of the hyperbolic tangent function is the dimensionless combination $\beta \sqrt{-\triangle}$.

The final result for renormalized free energy at finite temperature $\bar{F}_{\text {ren }}^{\beta}$ is presented by a sum of eqs. (19)-(21) and renormalized free energy at zero temperature $\bar{F}_{\text {ren }}^{\infty}$. After the zeta regularization (12), the latter one takes the form,

$$
\begin{aligned}
\bar{F}_{\text {ren }}^{\infty}= & -\frac{1}{32 \pi^{2}} \int \mathrm{d}^{3} x \bar{g}^{1 / 2}\left\{\bar{R}_{i j} \gamma_{1}(-\triangle) \bar{R}^{i j}+\bar{R} \gamma_{2}(-\triangle) \bar{R}\right. \\
& \left.+\bar{P} \gamma_{3}(-\triangle) \bar{R}+\bar{P} \gamma_{4}(-\triangle) \bar{P}+\mathrm{O}\left[\Re^{3}\right]\right\} .
\end{aligned}
$$

where form factors $\gamma_{i}(-\triangle), i=1, \ldots 4$, are

$$
\begin{aligned}
& \gamma_{1}(-\triangle)=\frac{1}{60}\left[-\ln \left(-\frac{\triangle}{\mu^{2}}\right)+\frac{46}{15}\right], \quad \gamma_{3}(-\triangle)=-\frac{1}{18} \\
& \gamma_{2}(-\triangle)=\frac{1}{180}\left[\ln \left(-\frac{\triangle}{\mu^{2}}\right)-\frac{97}{30}\right], \quad \gamma_{4}(-\triangle)=\frac{1}{2}\left[-\ln \left(-\frac{\triangle}{\mu^{2}}\right)+2\right] .
\end{aligned}
$$

\section{High temperature expansion of free energy}

Let us emphasize that the formulae (19)-(21) are valid at arbitrary finite temperature. Unfortunately direct evaluation of integrals (20) is possible only if one studies asymptotic behavior of the free energy, e.g., high temperature regime. It can be done relatively easy, because, as usual, the problem boils down to finding $\beta \rightarrow 0$ asymptotic of the thermal form factors (20)-(21). One has to be careful while dealing with mutually compensating singularities in $\gamma_{i}^{\beta}$. After some algebra, the outcome for, e.g. (9), is,

$$
\begin{aligned}
\gamma^{\beta}(-\triangle)= & \frac{2 \pi^{2}}{\beta \sqrt{-\triangle}}+2\left[\ln \left(\frac{\beta \sqrt{-\triangle}}{4 \pi}\right)-1+\mathrm{C}\right] \\
& -\frac{\zeta(3)}{24 \pi^{2}} \beta^{2}(-\triangle)+\frac{\zeta(5)}{640 \pi^{4}} \beta^{4}(-\triangle)^{2}+\mathrm{O}\left[\beta^{6}\right], \quad \beta \rightarrow 0,
\end{aligned}
$$


where $\mathrm{C}$ is the Euler constant and $\zeta$ is the Riemann zeta function.

Having (19) expanded in temperature series, we can add the vacuum free energy (22) to it. The result for the high temperature expansion of the renormalized one loop free energy takes a form,

$$
\begin{aligned}
\bar{F}_{\text {ren }}^{\beta}= & -\int \mathrm{d}^{3} x \bar{g}^{1 / 2}\left\{\frac{\pi^{2}}{90 \beta^{4}}+\frac{1}{24 \beta^{2}} \bar{P}\right. \\
& +\frac{1}{32 \beta}\left[\frac{1}{16} \bar{R}_{i j} \frac{1}{\sqrt{-\triangle}} \bar{R}^{i j}-\frac{25}{1152} \bar{R} \frac{1}{\sqrt{-\triangle}} \bar{R}\right. \\
& \left.-\frac{1}{12} \bar{P} \frac{1}{\sqrt{-\triangle}} \bar{R}+\bar{P} \frac{1}{\sqrt{-\triangle}} \bar{P}\right] \\
& \left.+\frac{1}{16 \pi^{2}}\left(\ln \left(\frac{\beta \mu}{4 \pi}\right)+\mathrm{C}\right)\left[\frac{1}{60} \bar{R}_{\mu \nu} \bar{R}^{\mu \nu}-\frac{1}{180} \bar{R} \bar{R}+\frac{1}{2} \bar{P} \bar{P}\right]\right\} \\
& +\mathrm{O}\left[\bar{\Re}^{3}\right]+\mathrm{O}\left[\beta^{2}\right], \quad \beta \rightarrow 0 .
\end{aligned}
$$

First and foremost, one should note that $1 / \beta$ order has now form $\Re \frac{1}{\sqrt{-\triangle}} \Re$. Therefore it is essentially nonlocal, and techniques based on local short time expansions can not generate it. We expect that terms of higher orders in curvatures [12, 14] will also give nonlocal contribution to the linear $T$ order. The meaning of these structures can be understood from an elegant spectral representation [11, 12] in terms of massive Green functions,

$$
\frac{1}{\sqrt{-\triangle}}=\frac{2}{\pi} \int_{0}^{\infty} \mathrm{d} m \frac{1}{m^{2}-\triangle} .
$$

A remarkable property of the expression (25) is that all $\ln (-\triangle)$ have disappeared, leaving logarithmic temperature dependance in the form of $\ln (\beta \mu)$, the combination known in flat space thermal field theory [5, 19, 20].

Combination of quadratic in curvatures terms at the logarithm is nothing but the trace of the second Schwinger-DeWitt coefficient $a_{2}$, taken with Riemann curvature expressed via Ricci one [1], 2, 10]. Higher powers of $\beta$ (see eq. (24)), which we discarded in $\bar{F}_{\text {ren }}^{\beta}$, are also quadratic in curvatures parts of higher Schwinger-DeWitt coefficients [14].

All local terms in our result (25) perfectly reproduce those of ref. [18]. We also obtained the explicit form of all nonlocal terms of the second order in curvatures. They contain form factor $1 / \sqrt{-\triangle}$ and turn out to be proportional to $1 / \beta$. Implicitly these nonlocal parts are contained in the term denoted by $\bar{\zeta}_{(3)}^{\prime}(0)$ in eq. (17) of 18 .

\section{Conclusion}

The main result of this paper is the one loop nonlocal free energy for scalar fields at finite temperature, eqs. (19)-(23). Since in the setting of our problem $\bar{F}_{\beta}$ and $\bar{W}^{\beta}$ differ only by a factor $\beta^{-1}$, one can speak about free energy and effective action interchangeably. One can derive other quantities, such as Green function, stress tensor or entropy, by varying this free energy correspondingly with respect to the potential, metric or temperature. Clearly, to proceed to physical applications one first needs some kind of spectral representation for 
finite temperature form factors, like the ones derived for zero temperature effective action 9, 10, 11, 12 or its high temperature limit (26).

It should be emphasized that the perturbation theory we used to derive our results works well for highly inhomogeneous background fields, i.e., $\nabla \nabla \Re>>\Re^{2}$. Therefore, eqs. (19)(23), (25) are not applicable to the opposite case of constant or nearly constant background.

Our work also demonstrates, that infrared divergencies in finite temperature field theory are artificial and a correctly computed thermal effective action is infrared finite at all stages of calculations, what supports the main idea of ref. [16].

Generalization of our derivations to arbitrary static spaces does not seem to pose serious complications, and involves well elaborated techniques of conformal transformations to ultrastatic spacetimes [21, 18]. In order to include into consideration manifolds with boundaries, a major revision of the perturbation theory [10] is needed, though it is still feasible to do. It is also possible to include the chemical potential [7]. Non-Abelian gauge fields can be taken into consideration as well, but the factorization formula (7) will be modified by additional terms [22], connected to temporal components of gauge fields. One can also easily generalize the results to quantum fields of other spins.

\section{Acknowledgments}

This work was partially supported by National Science and Engineering Research Council of Canada. Yu. G. is supported also by National Research Fellowships from Canadian Institute for Theoretical Astrophysics, and A. Z. is grateful to the Killam Trust for its financial support. 


\section{References}

[1] B. S. DeWitt, Dynamical theory of groups and fields (Gordon and Breach, New York, 1965).

[2] A. O. Barvinsky and G. A. Vilkovisky, Phys. Rep. 119 (1985) 1.

[3] E. S. Fradkin, Dokl. Akad. Nauk SSSR 125 (1959) 313; Nucl. Phys. 12 (1959) 465.

[4] P. C. Martin and J. Schwinger, Phys. Rev. 115 (1959) 1342.

[5] L. Dolan and R. Jackiw, Phys. Rev. D 9 (1974) 3320.

[6] J. S. Dowker and R. Critchley, Phys. Rev. D 15 (1977) 1484.

[7] J. S. Dowker and J. P. Schofield, Nucl. Phys. B 327 (1989) 267.

[8] A. O. Barvinsky, V. P. Frolov and A. I. Zelnikov, Phys. Rev. D 51 (1995) 1741.

[9] A. O. Barvinsky and G. A. Vilkovisky, Nucl. Phys. B 282 (1987) 163.

[10] A. O. Barvinsky and G. A. Vilkovisky, Nucl. Phys. B 333 (1990) 471.

[11] A. O. Barvinsky and G. A. Vilkovisky, Nucl. Phys. B 333 (1990) 512.

[12] A. O. Barvinsky, Yu. V. Gusev, G. A. Vilkovisky, and V. V. Zhytnikov, Report of the University of Manitoba (1993), unpublished.

[13] G. A. Vilkovisky, in Quantum theory of gravity, ed. S. M. Christensen (Hilger, Bristol, 1984) p. 169.

[14] A. O. Barvinsky, Yu. V. Gusev, G. A. Vilkovisky, and V. V. Zhytnikov, J. Math. Phys. 35 (1994) 3543.

[15] A. O. Barvinsky, Yu. V. Gusev, G. A. Vilkovisky, and V. V. Zhytnikov, Nucl. Phys. B 439 (1995) 561.

[16] I. T. Drummond, P. R. Horgan, P. V. Landshoff, and A. Rebhan, Phys. Lett. B 398 (1997) 326.

[17] J. S. Dowker and G. Kennedy, J. Phys. A 11 (1978) 895.

[18] J. S. Dowker and J. P. Schofield, Phys. Rev. D 38 (1988) 3327.

[19] H. E. Haber and H. A. Weldon, Phys. Rev. D 25 (1982) 502.

[20] F. T. Brandt and J. Frenkel, Phys. Rev. D 55 (1997) 7808.

[21] J. S. Dowker, Phys. Rev. D 33 (1986) 3150.

[22] A. V. Leonidov and A. I. Zelnikov, Phys. Lett. B 276 (1992) 122. 